\title{
Lithium-Ion Supercapacitor Using Vertically-aligned Carbon Nanotubes from Direct Growth Technique, and its Electrochemical Characteristics
}

\author{
Mohd A. Azam ${ }^{a, *}$, Nor S. A. Manaf ${ }^{a}$, Qumrul Ahsan ${ }^{a}$, \\ Oskar H. Hassan ${ }^{b}$ and Muhd Z. A. Yahya ${ }^{c}$ \\ ${ }^{a}$ Carbon Research Technology Research Group, Advanced Manufacturing Centre, \\ Faculty of Manufacturing Engineering, Universiti Teknikal Malaysia Melaka, \\ Hang Tuah Jaya, 76100 Durian Tunggal, Melaka, Malaysia \\ ${ }^{b}$ Department of Industrial Ceramic, Faculty of Art and Design, Universiti Teknologi MARA, \\ 40450 Shah Alam, Selangor, Malaysia \\ ${ }^{c}$ National Defence University of Malaysia, Kem Sungai Besi, 57000 Kuala Lumpur, Malaysia
}

Received July 25, 2017; acepted December 25, 2017

\begin{abstract}
This paper reports the fabrication of a lithium ion supercapacitor from vertically-aligned carbon nanotubes (VACNTs) directly grown on a conductive substrate (SUS 310S alloy), using alcohol catalytic chemical vapour deposition technique. CNTs direct growth technique on an electrically conducting foil simplifies the electrode assembly, thus reducing the fabrication process, because the foil can directly act as a current collector. With the VACNT direct growth technique, the supercapacitor electrode was easily prepared and assembled with a non-aqueous $1 \mathrm{M} \mathrm{LiPF}_{6}$ electrolyte. Experimental results show that CNTs (multi-walled type structures of good quality) were perpendicularly grown to the substrate. This device demonstrates a specific capacitance of up to $101 \mathrm{~F} \mathrm{~g}^{-1}$ (at a scan rate of $1 \mathrm{mVs}^{-1}$ ), and a high-rate capability, up to a scan rate of $1000 \mathrm{mVs}^{-1}$. The VACNT electrode electrochemical performance was also measured by galvanostatic charge-discharge and electrochemical impedance spectroscopy. The effect of free standing CNTs direct growth on the current collector makes insulating binder material unnecessary, thus producing better ion accessibilities to its surface. This also contributes to the good and reliable electrochemical supercapacitor performance.
\end{abstract}

Keywords: vertically-aligned CNT; alcohol catalytic CVD; direct growth technique and Li- ion supercapacitor.

\section{Introduction}

In modern engineering, electrochemical capacitors (EC), also known as electrochemical double layer capacitors (EDLC) or supercapacitors, are able to store electrical charge in a growing variety of applications in many fields [1], such as portable electronics, digital communications, hybrid electric vehicles, etc.

\footnotetext{
*Corresponding author. E-mail address: asyadi@utem.edu.my
} 
[2]. EC, including lithium (Li)-ion capacitor (LIC), fills the critical performance gap between batteries high energy density and conventional capacitors high power density [3]. ECs' main advantages are that they can provide high power density, high energy density, long life cycle and fast charge-discharge rate $[4,5$, 6 and 7]. EDLC's energy storage mechanism arises from electrostatic charge or physical separation at the electrode-electrolyte interface [8]. Pseudocapacitor charge storage ability depends on the fast redox reactions [9].

Nowadays, nanostructured carbon materials, such as CNTs, have been focused as a highly potential electrode material for EC, due to their remarkable electrical charge storage ability, large specific surface area, chemical stability, and excellent electrical conductivity [10 and 11]. Nowadays, researchers are focusing on the vertically aligned CNTs (VACNTs) as an electrode material, due to their advantages for specific surface areas, and to the ion accessibility that is more efficient and better, compared to entangled CNT [12]. Alcohol catalytic chemical vapour deposition (ACCVD) is a promising technique to produce VACNTs, being well-known for its economical merit, wide selectivity of substrates, and highly yielding catalytic reaction for CNTs growth [13]. The used alcohol, typically ethanol $\left(\mathrm{C}_{2} \mathrm{H}_{5} \mathrm{OH}\right)$-grown nanotubes, is nearly free from any amorphous carbon, due to the $\mathrm{OH}^{-}$radical etching action [14].

In addition, the electrode preparation technique is another critical issue of EC electrochemical performance [15]. In the conventional electrode preparation technique, such as in activated carbon or entangled CNTs, the electrode might have large internal resistance, due to the inclusion of insulating binder or adhesive materials, which become barriers in the access paths for electrons and electrolyte ions [16]. Hence, direct growth technique of aligned CNTs on a conducting substrate is an attractive electrode preparation technique, due to the direct electrical contact between the VACNTs electrode and the current collector. The conducting substrate can directly act as current collector, simplifying the conventional electrode assembly process. Thereby, this technique could reduce equivalent series resistance (ESR), and enhance capacitance performance [17].

Herein, the primary goal was to work on the direct growth of VACNTs metallic alloy (SUS 310S foil) substrates, using ACCVD technique to fabricate the supercapacitor electrode. The supercapacitor was constructed from the as-grown VACNT electrodes and a non-aqueous $1 \mathrm{M}$ lithium hexafluorophosphate $\left(\mathrm{LiPF}_{6}\right)$ electrolyte. The as-grown (AG)-VACNT electrode samples' morphological and structural properties were examined by electron microscopic and Raman spectroscopy techniques. The secondary goal was to determine the VACNT electrode electrochemical properties in a Li-ion electrolyte, from the viewpoint of cycling behaviors, charge discharge ability and frequency response analysis.

\section{Experimental}

\section{Preparation of VACNT electrodes}

For the conducting substrate to be used as current collector, SUS 310S foils with $0.2 \mathrm{~mm}$ thickness were cut into $15 \mathrm{~mm}$ diameter round substrates. The average weight for two SUS $310 \mathrm{~S}$ foils with this dimension is $0.2761 \mathrm{~g}$. Thin films of Al 
(25 nm) and, subsequently, of Co (7-9 nm) were deposited on the SUS 310S foils, using radio frequency (RF) magnetron sputtering deposition. After the thin films deposition, the sample weight (two thin films) was measured; the average weight of thin films (Co/Al-O) was $0.0060 \mathrm{~g}$. For the formation of thermallyoxidized aluminium oxide (Al-O), the substrate was transferred to the CVD furnace (RTP-1000D4), and baked at $500{ }^{\circ} \mathrm{C}$ in static air, for $10 \mathrm{~min}$, just before the CVD process. As a pre-treatment, argon gas was purged at a pressure of $350 \mathrm{mTorr}$, concurrently with a $5 \mathrm{~min}$ rapid heating up to CVD temperature of $725^{\circ} \mathrm{C}$. The argon gas was continuously flowed for another $5 \mathrm{~min}$ of the annealing process. For CNT growth, the CVD processing parameter was fixed at $725^{\circ} \mathrm{C}$, with ethanol flow rates of $100-130 \mathrm{sccm}, 10-15$ Torr internal pressure, and 15 min CVD processing time. The samples weight was again measured after the CVD process, and the average weight of as-grown VACNT/ Co/Al-O was $0.0062 \mathrm{~g}$. Therefore, CNTs' net mass loading, as an active material (0.0002 $\mathrm{g}$ or $0.20 \mathrm{mg}$ ), was obtained by subtraction of the foil weight increase, with only $\mathrm{Co} / \mathrm{Al}-\mathrm{O}$ films, from that with CNTs.

A field emission scanning electron microscope (FESEM, $20 \mathrm{kV}$ ) and a transmission electron microscope (TEM, $120 \mathrm{kV}$ ) were used to characterize the AG-VACNTs morphology. Raman spectroscopy was used to further investigate the VACNTs structural and graphitic properties, using the $632.8 \mathrm{~nm}$ wavelength laser excitation: a HeNe laser.

\section{Electrochemical measurements}

The supercapacitor was build with symmetric AG-VACNT electrodes cells, separated by $25-\mu \mathrm{m}$ polypropylene (PP; Tonen) and a $1 \mathrm{M} \mathrm{LiPF}_{6}$ solution as electrolyte. The capacitor was assembled using a battery jig (Takumi Giken), as shown in Fig. 1.

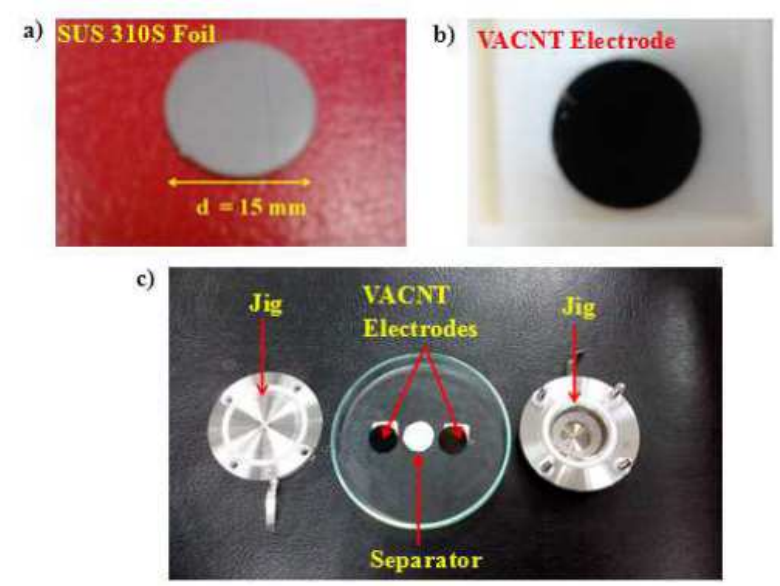

Figure 1. Construction of a Li-ion capacitor using VACNT electrodes. (a) digital image of the blank SUS 310S foil, (b) digital image of grown VACNTs on a SUS 310S foil, and (c) VACNT electrode, and the components for the supercapacitor cell.

Cell assembly was carried out in a glove box, with controlled oxygen and moisture concentration. All electrochemical measurements were carried out at room temperature $\left(25^{\circ} \mathrm{C}\right)$. 
$\mathrm{CV}$ and galvanostatic charge-discharge measurements were performed using Wonatech: WBCS3000 potentiostat/galvanostat. CV measurement was conducted to measure the electrode cyclic behaviour, as well as the specific gravimetric capacitance $\left(\mathrm{C}_{\mathrm{sp}}\right)$. The potential was set from 0.0 to $3.0 \mathrm{~V}$, due to the Li electrolyte's thermodynamic window, which can actually be up to $4.6 \mathrm{~V}$. The scan rate was set to $1,5,10,50,100,500$, and $1000 \mathrm{mV} \mathrm{s}^{-1}$. In addition, chargedischarge measurements were conducted to measure the charging and discharging behaviour with the different applied currents, namely 1, 5 and 10 $\mathrm{mA}$. The EIS measurement was then conducted using a frequency response analyzer (Metrohm Autolab), ranging from $100 \mathrm{KHz}$ to $0.01 \mathrm{~Hz}$, at the amplitude of $10 \mathrm{mV}$.

\section{Results and discussion}

\section{Morphological and structural properties of the VACNT electrode}

Fig. 2 shows FESEM images of VACNTs that were directly grown on a SUS $310 \mathrm{~S}$ foil.
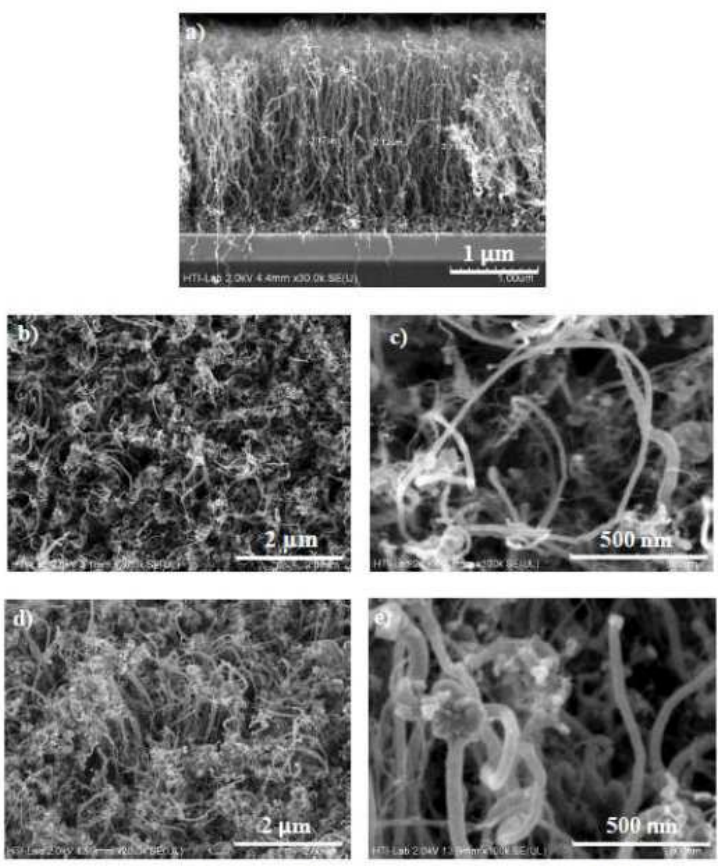

Figure 2. FESEM images of VACNTs directly grown on a SUS 310S foil. (a) crosssection view, (b) top view (low magnification), (c) top view (high magnification), (d) $45^{\circ}$ tilted (low magnification), and (e) $45^{\circ}$ tilted (high magnification).

Observations were directly made from the above substrate, at a tilt angle of $45^{\circ}$, and cross-sectionally, to confirm the vertical structure of the CNTs forest on the substrates. The CNTs were found to be vertical aligned, perpendicular to the substrate, where most of the substrate's area was covered by VACNTs (Fig. 2 (a)). From the cross section image, the VACNTs height is approximately 2.10$\mu \mathrm{m}$. It was experimentally proved that the CVD-grown VACNTs height can increase with the extended CVD processing time, and some adjustments on other 
parameters. In this work, and with the present CVD system setup, 15 min could be a short time. However, the effect of extended CVD processing time (i.e., more than $30 \mathrm{~min}$ ) might also damage the VACNTs structure.

Meanwhile, TEM image in Fig. 3 reveals that the VACNT diameter grown by ACCVD technique varies from 2 to $20 \mathrm{~nm}$, and that the VACNTs grown on the SUS $310 \mathrm{~S}$ foil are multi-walled CNTs. There are also some Co particles in the TEM image, which may be the cobalt catalyst that maintains the CNTs growth.

The VACNTs were catalytically grown on SUS 310S foils from a Co catalyst and catalyst-supports, respectively. Thermally oxidized Al-O, used as a catalystsupport layer, plays an important role in CNTs vertical growth, due to its pore structures and roughened surface [12]. The porous structure of the metal oxide layer will trap catalyst nanoparticles during the annealing process, and produce strong adhesion force, due to the obtuse contact angle amid them. During CNT growth, the metal oxide layer will act as a core to support CNT alignment, and simultaneously prevent the catalyst nanoparticle lifts off the substrate, during initial hydrocarbon decomposition and carbon diffusion [18]. The Al-O layer also supposedly prevents catalyst nanoparticles aggregation [11].Therefore, Al-O used as buffer layers will enhance the aligned CNT growth efficiency [19]. It is also important to note that the CNT diameter varied due to some nonuniformities of the catalyst thin film evolution during the CVD annealing process.
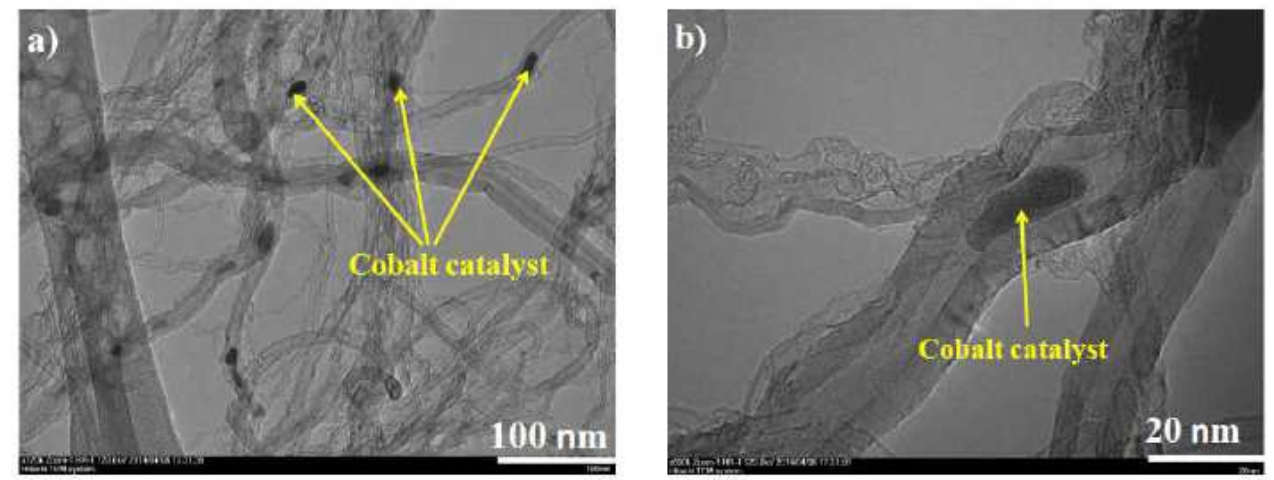

Figure 3. TEM images of VACNTs after being cut from the SUS foil, where Co catalyst particles can be observed inside, and at the edge of the CNTs. (a) lower magnification, and (b) higher magnification.

Raman spectroscopy is a useful non-destructive method for the structural characterization of different carbon materials [20]. From Raman spectroscopy analysis, CNTs quality is according from G-band to D-band peak intensity ratio, $\mathrm{I}_{\mathrm{G}} / \mathrm{I}_{\mathrm{D}}$. Large G-band to D-band intensity ratio generally corresponds to CNTs of high quality [21]. From Raman spectra measured by $633 \mathrm{~nm}$ laser excitation (Fig. $4)$, the intensity ratio $\left(I_{G} / I_{D}\right)$ was found to be 2.70 . The Raman spectra also exhibit peaks of the radial breathing mode (RBM) from 100 to $280 \mathrm{~cm}^{-1}$, which reveals the presence of single-walled CNTs, a D-band (defect, amorphous carbon) at approximately $1324 \mathrm{~cm}^{-1}$, and a G-band (graphite, CNTs) at approximately $1590 \mathrm{~cm}^{-1}$. The peak at $2606 \mathrm{~cm}^{-1}$ represents the $2 \mathrm{D}$-band peak, which can indicate the presence of graphene. However, we should note that CNT 
has the structure of a rolled-up graphene sheet, which explains the 2D-band peak appearance. High G-band intensity confirms the fact that CNTs are dominantly grown. Also, it is possible that some of the CNTs were opened up (broken), and that the Raman laser has detected that particular graphene sheet/film. Raman spectra analysis also showed that VACNTs were common or dominant in multiwalled CNTs. This result was in good agreement with the TEM observation, and the low peak of RBM region. Therefore, this confirmed that CNTs could also effectively be grown on metal alloys, using ACCVD system.

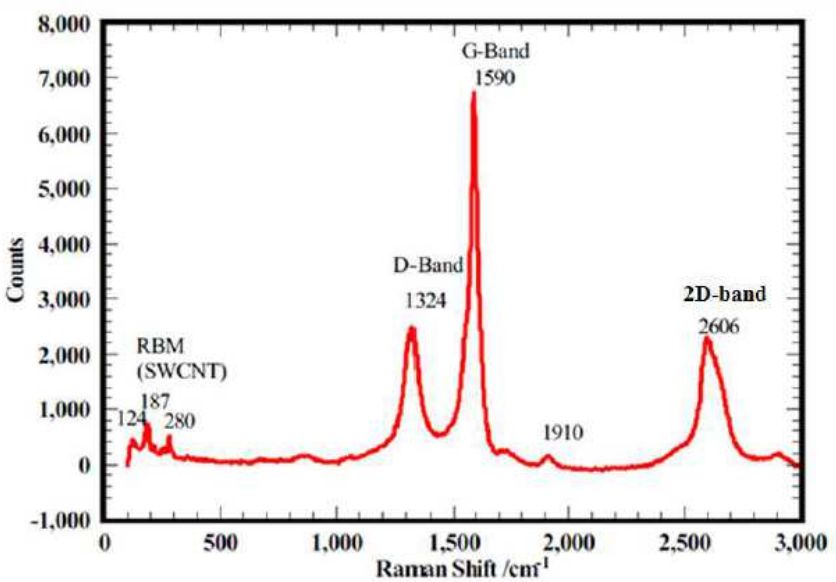

Figure 4. Structural and graphitic properties of VACNTs directly grown from Co/AlO/SUS $310 \mathrm{~S}$, confirming a relatively good quality of the CNTs

\section{Electrochemical performances of the Li-ion capacitor}

The performance and charge storage behaviour of VACNTs electrode in a $1 \mathrm{M}$ $\mathrm{LiPF}_{6}$ electrolyte were investigated by $\mathrm{CV}$ analysis at various scan rates, using 1 , 5, 10, 50, 100, 500 and $1000 \mathrm{mV} \mathrm{s}^{-1}$ scan rates (Fig. 5). The Li-ion electrolyte, which acts as an electric current carrier, is another important factor in the electrochemical performance enhancement [22]. In this work, $\mathrm{LiPF}_{6}$ was used as an electrolyte, with the potential window range from 0.0 to $3.0 \mathrm{~V}$. From the $\mathrm{CV}$ measurement with various scan rates, it was confirmed that the VACNT electrode has double-layer capacitance behaviour, when used as an electrode material in ECs application. At $1 \mathrm{mV} \mathrm{s}^{-1}$ of CV scan rate, the calculated $\mathrm{C}_{\mathrm{sp}}$ was $101 \mathrm{Fg}^{-1}\left(0.0-3.0 \mathrm{~V}\right.$ potential). The $\mathrm{C}_{\mathrm{sp}}$ of the VACNT electrode was calculated from equation 1 , using the recorded CVs at a scan rate of 1, 5, 10, 50, 100, 500 and $1000 \mathrm{mV} \mathrm{s}^{-1}$.

$$
C_{s p}=\left(\int_{E_{1}}^{E_{2}} i(E) d E\right) \div 2\left(E_{2}-E_{1}\right) m v
$$

where $E_{1}$ and $E_{2}$ are the cutoff potentials in $C V, i(E)$ is the current and the total voltammetric charges obtained by integration of positive (charge) and negative (discharge) sweeps in $\mathrm{CV},\left(\mathrm{E}_{2}-\mathrm{E}_{1}\right)$ is the cell potential window width, $\mathrm{m}$ is the CNT mass per electrode, and $v$ is the scan rate [23]. In our previous work in reference [23], we have discussed that VACNTs optimum height is critical for a supercapacitor to have a good performance. VACNTs optimum height must not 
exceed $60-\mu \mathrm{m}$. Thus, a $2-\mu \mathrm{m}$ height of VACNT forest is considered acceptable to achieve its maximum capacitance value during the device characterization.

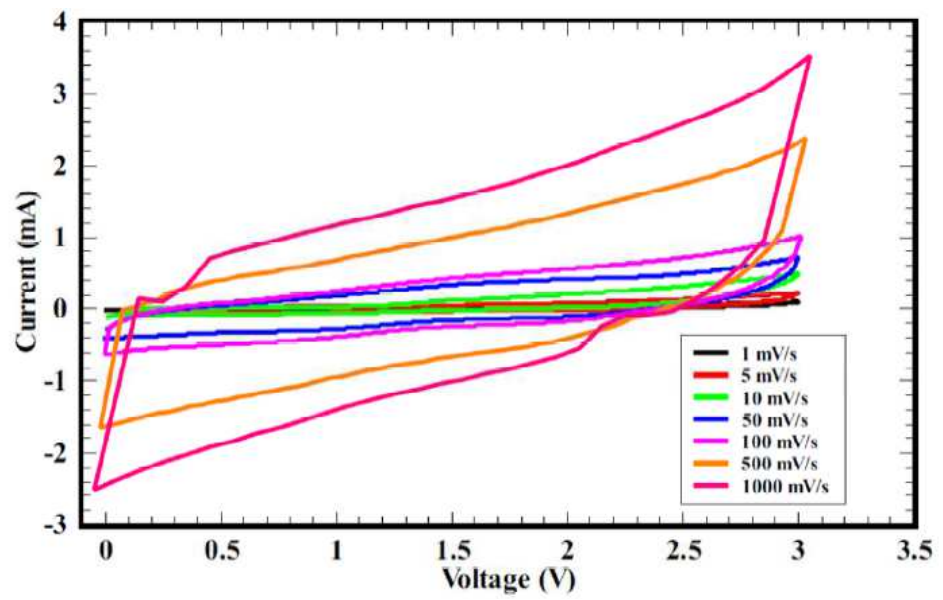

Figure 5. Charge-discharge performance of VACNT electrode cooperated with Li-ion electrolyte from the $\mathrm{CV}$ curves measured at various scan rates $(1,5,10,50,100,500$, and $1000 \mathrm{mV} \mathrm{s}^{-1}$ ).

The CV curves also show near rectangular shapes, even at the scan rate of 1000 $\mathrm{mV} \mathrm{s} \mathrm{s}^{-1}$, with no redox peak, indicating a supercapacitive behaviour, and becoming the typical shape of EDLC. This result also confirms that the AGVACNT electrodes have high reversibility and high power density [24 and 25]. These significant results from $\mathrm{C}_{\mathrm{sp}}$ value and $\mathrm{CV}$ curve shape revealed that the AG-VACNTs electrode had good electrical contact with the SUS 310S substrate. $\mathrm{CV}$ near rectangular shape performed at high scan rates of $1000 \mathrm{mV} \mathrm{s}^{-1}$, and showed a typical ideal capacitive charging and discharging characteristic across the potential range from 0.0 to $3.0 \mathrm{~V}$, suggesting that the integrated VACNTcurrent collector systems have very rapid current response on voltage reversal with low equivalent series resistance (ESR) [17 and 20].

The VACNT electrode performance in $1 \mathrm{M} \mathrm{LiPF}_{6}$ was further evaluated using galvanostatic charge-discharge analysis at 1, 5, and $10 \mathrm{~mA}$ (Fig. 6 (a), (b), (c)), from 0.0 to $3.0 \mathrm{~V}$, and back to $0.0 \mathrm{~V}$. It was continuously measured up (but not limited) to 10 cycles, and was very stable in terms of charging and discharging behaviour.

Various constant currents were applied to observe the ability of the electrodes to work from lower to higher current densities. The results showed only small IR (ohmic) drop when using $1 \mathrm{~mA}$ current, while IR drop at $5 \mathrm{~mA}$ and $10 \mathrm{~mA}$ was not obvious at all. This indicates very small internal resistance and excellent power characteristics of the electrode. All the charge-discharge curves for this supercapacitor are near triangular in shape, and without significant IR drop, indicating that the electrode has ideal capacitive charging-discharging behaviour, with less faradaic reaction and excellent electrochemical reversibility [26 - 28]. The success of the working device neglects the effect of the Co nanoparticles that incorporated the as-grown CNTs. 

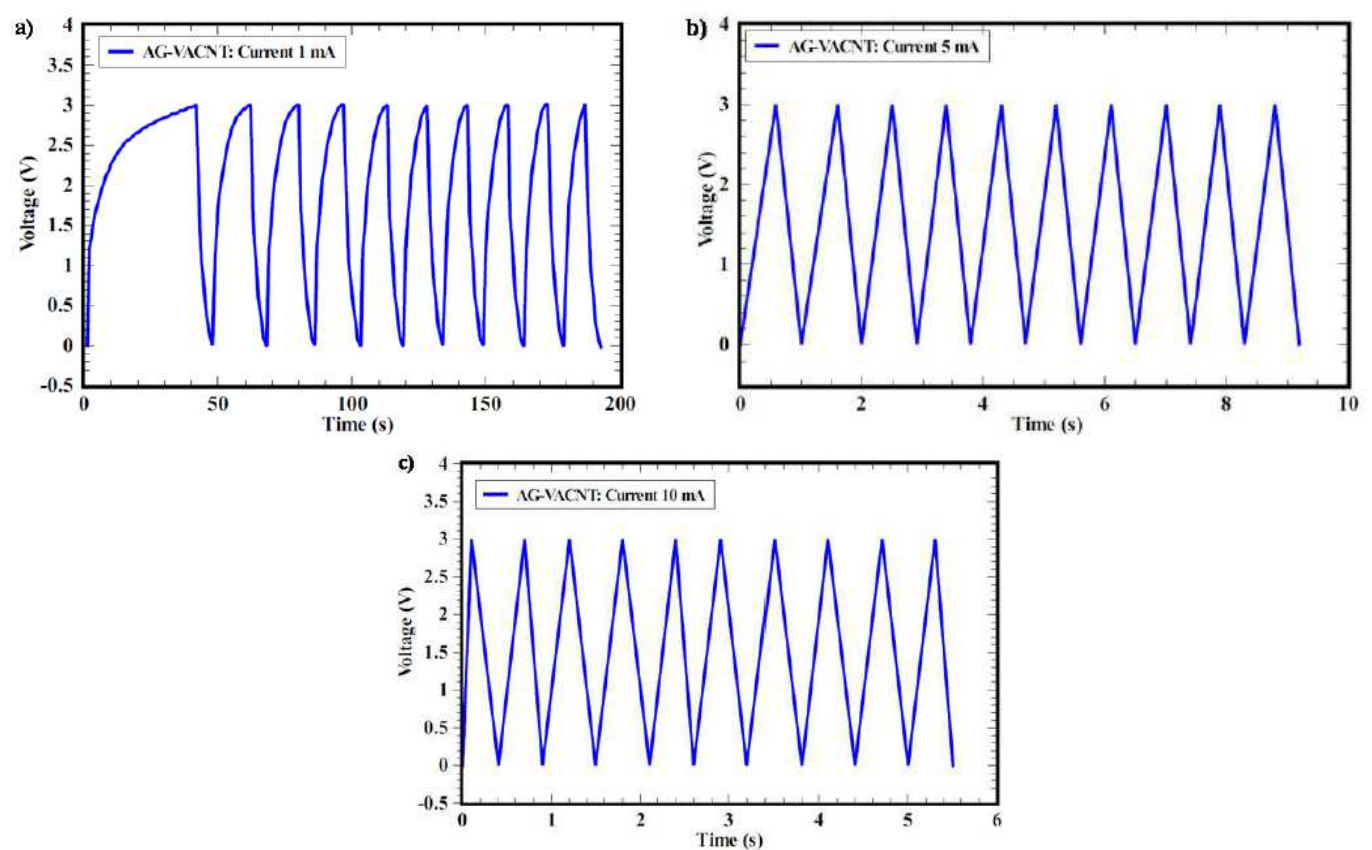

Figure 6. Charge discharge behaviour of the Li-ion capacitor measured at different applied currents. (a) $1 \mathrm{~mA}$, (b) $5 \mathrm{~mA}$, and (c) $10 \mathrm{~mA}$.

Small and/or no IR drop for this capacitor show direct connection between the aligned CNT electrode and the current collector, accelerating the ion transport for the charge storage mechanism. The charge-discharge curve showed that the EC had very rapid charge-discharge performance (in second), which is a well-known EC advantage. On the other hand, the charge-discharge cycle duration was found to decrease with increasing current. The results show that the charge-discharge duration for 10 cycles was $190 \mathrm{~s}, 9.2 \mathrm{~s}$ and $5.5 \mathrm{~s}$, at different $1 \mathrm{~mA}, 5 \mathrm{~mA}$, and 10 $\mathrm{mA}$ applied currents, respectively. At low current, the charging/discharging process took more time, which is attributed to the sufficient insertion or release of electrolyte ions [29].

Further evaluation of the VACNT electrode's electrochemical properties was done using EIS analysis. EIS is a valuable technique to investigate energy devices' resistivity performance, such as charge transfer and electrode resistance. Fig. 7(a) illustrates the Nyquist plot of VACNT electrodes in a $1 \mathrm{M} \mathrm{LiPF}_{6}$ electrolyte. The inset shows the spectrum enlargement in high frequency region. The impedance behaviour was composed of three major processes occurred in the high, medium and low frequency regions, respectively [30]. Theoretically, features of a semicircle over the high frequency region, and a vertical line parallel to the low frequency range, show an ideal Nyquist plot [29].

Nyquist plots show almost vertical lines parallel to the imaginary axis in the low frequency region, which identifies an ideal capacitive behaviour of the VACNTs electrode, with a relatively low ESR value. This indicates that the CNT supercapacitors behave like pure capacitors at low frequencies [26]. At higher frequencies (inset of Fig. 7 (a)), the presence of a small semicircular shape of the curve represents the time constant related to the electrical charge transfer process at the electrode/electrolyte interface, or the diffusion of electrolyte ions to the VACNTs electrode. Also, the arc in the high frequency part is probably due to 
the VACNTs direct contact with the substrate [17]. The electrolyte resistance $\left(\mathrm{R}_{\mathrm{el}}\right)$ and the charge transfer resistance $\left(\mathrm{R}_{\mathrm{ct}}\right)$ measured from EIS data were found to be $4.5 \Omega$ and $8.6 \Omega$, respectively. The semicircle diameter $\left(R_{c t}-R_{e l}\right)$ represents ESR value [31]. ESR value for this AG-VACNT was found to be $4.1 \Omega$.

$\mathrm{Li}^{+}$ions from $\mathrm{LiPF}_{6}$ electrolyte started to diffuse at $2683 \mathrm{~Hz}(8.6 \Omega)$, and the diffusion stopped at $373 \mathrm{~Hz}(10 \Omega)$, where the maximum capacitance was reached and became dominant. The AG-VACNTs electrode also possessed a frequency dependent phase angle around $74^{\circ}$ (Figure 7 (b)). Therefore, the results strongly suggest that the directly grown VACNTs on the SUS 310S foils possessed low device resistivity, due to good electrical and ionic conductivity. This also indicates that the electron and electrolyte ions easily diffused and/or moved through the VACNTs electrode. The excellent performance highlights the important role of the VACNTs in storing and accumulating charge, thereby boosting both power density and energy density [32].
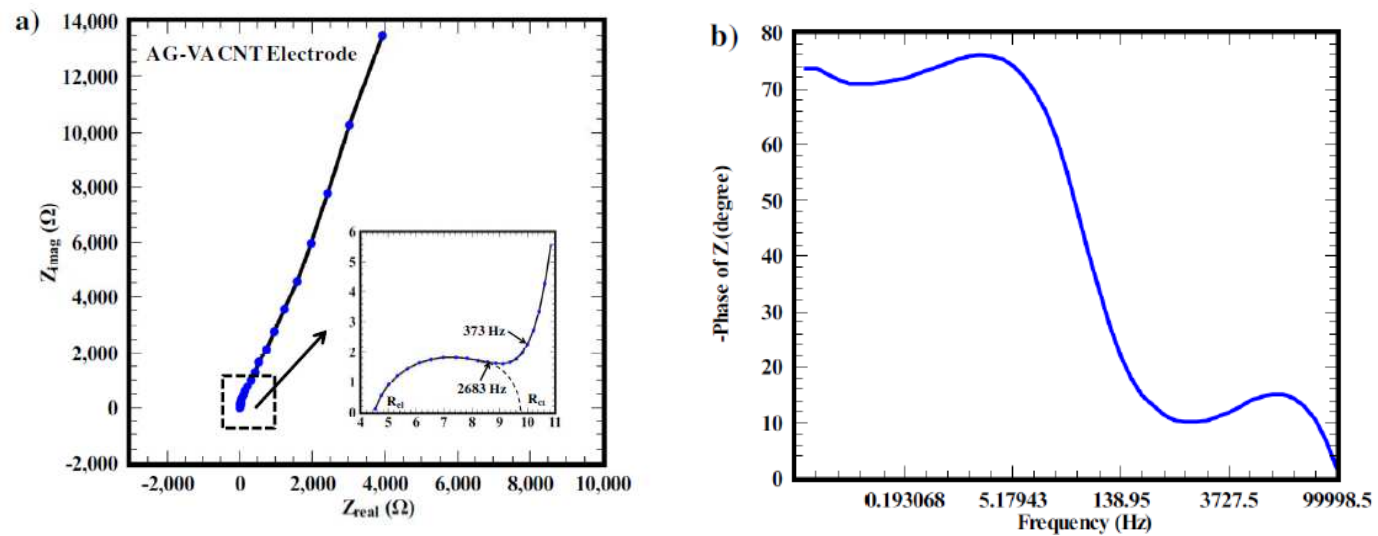

Figure 7. VACNT frequency response analysis in a $\mathrm{LiPF}_{6}$ electrolyte. (a) Nyquist plot showing the imaginary versus real part of impedance. The inset is the enlargement of the plot in the high frequency region, and (b) Bode plot of the supercapacitor with a frequency dependent phase angle of $74^{\circ}$.

\section{Conclusions}

A lithium-ion supercapacitor was fabricated from vertically-aligned multi-walled carbon nanotube (VA-MWCNT) electrodes, and $1 \mathrm{M} \mathrm{LiPF}_{6}$ as an electrolyte. From the electron microscopy observations, CNTs have a diameter in the range of 2 to $20 \mathrm{~nm}$, and a height of approximately $2.10-\mu \mathrm{m}$. On this type of symmetric capacitor electrode, CV analysis showed the typical rectangular shaped curves, suggesting the double layer capacitance behaviour of the electrode. This capacitor has a maximum gravimetric capacitance of $101 \mathrm{~F} \mathrm{~g}^{-1}$, calculated from the $\mathrm{CV}$ curve at a scan rate of $1 \mathrm{mV} \mathrm{s}^{-1}$. During charge-discharge measurements, a small IR drop was confirmed, and a near triangular shape of the curve indicates low series resistance and a high power characteristic. This was consistent with the EIS Nyquist plot that exhibited a close to vertical line along the imaginary axis, which again revealed the ideal capacitive properties of directly-grown CNTs on the conducting substrate. 
Additionally, further advancements on preparing aligned CNT electrodes are highly desired. For example, research on the optimum vertical height of the CNTs, and the usage of nano imprinting technique, to design the catalyst films/particles display for a consistent arrangement of the aligned CNTs. By this, ion accessibility can be improved, which could be another interesting feature to explore, for higher energy density and power density devices.

\section{Acknowledgements}

This work was financially supported by the eScienceFund research grant from Ministry of Science, Technology and Innovation (MOSTI), Malaysia No.: 03-0114-SF006/L00015. We also thank Universiti Teknikal Malaysia Melaka for the experimental facilities support.

\section{References}

1. Zhang $\mathrm{Y}$, Feng $\mathrm{H}, \mathrm{Wu} \mathrm{X}$, et al. Progress of electrochemical capacitor electrode materials: A review. Int J Hydrogen Energy. 2011;34:4889-4899.

2. Li J, Cheng X, Shashurin A, et al. Review of Electrochemical Capacitors Based on Carbon Nanotubes and Graphene. Graphene. 2012;1:1-13.

3. Simon P, Gogotsi Y. Charge storage mechanism in nanoporous carbons and its consequence for electrical double layer capacitors. Philos. Trans. A. Math. Phys. Eng. Sci. 2010;368:3457.

4. Emmenegger C, Mauron P, Sudan P, et al. Investigation of electrochemical double-layer capacitors electrodes based on carbon nanotubes and activated carbon materials. J Power Sources. 2003;124:321-329.

5. He X, Jiang L, Yan S, et al. Direct synthesis of porous carbon nanotubes and its performance as conducting material of supercapacitor electrode. Diam Relat Mater. 2008;17:993-998.

6. Hsieh CT, Hsu SM, Lin JY. Fabrication of Graphene-Based Electrochemical Capacitors. Jpn J Appl Phys. 2012;51:01AH06.

7. Shen J, Liu A, Tu Y, et al. How carboxylic groups improve the performance of single-walled carbon nanotube electrochemical capacitors. Energy Environ Sci. 2011;4:4220.

8. Gourdin G, Jiang T, Smith P, et al. The effects of cell assembly compression on the performance of carbon electrochemical double-layer capacitor electrodes. J Power Sources. 2012;215:179-187.

9. Candelaria SL, Shao Y, Zhou W, et al. Nanostructured carbon for energy storage and conversion. Nano Energy. 2012;1:195-220.

10. Inagaki M, Konno $\mathrm{H}$, Tanaike O. Carbon materials for electrochemical capacitors. J. Power Sources. 2010;195:7880-7903.

11. Kim BW, Chung HG, B. Min K, et al. Electrochemical Capacitors Based on Aligned Carbon Nanotubes Directly Synthesized on Tantalum Substrates. Bull Korean Chem Soc. 2010;31:3697-3702. 
12. Azam MA, Manaf NSA, E. Talib E, et al. Aligned carbon nanotube from catalytic chemical vapor deposition technique for energy storage device, a review. Ionics. 2013;19:1455-1476.

13. Azam MA, Abd Rashid MW, Isomura K, et al. X-Ray and Morphological Characterization of Al-O Thin Films Used for Vertically Aligned SingleWalled Carbon Nanotube Growth. Adv Mater Res. 2012;620:213-218.

14. Bistamam MSA, Azam MA, Manaf NSA, et al. An Overview of Selected Catalytic Chemical Vapor Deposition Parameter for Aligned Carbon Nanotube Growth. Nanosci Nanotechnol-Asia. 2014;4:2-30.

15. Manaf NSA, Bistamam MSA, Azam MA. Development of High Performance Electrochemical Capacitor: A Systematic Review of Electrode Fabrication Technique Based on Different Carbon Materials. ECS J Solid State Sci Technol. 2013;2:M3101-M3119.

16. Azam MA, Dorah N, Seman RNAR, et al. Electrochemical performance of activated carbon and graphene based supercapacitor. Mater Technol. 2014;30:A14-A17.

17. Shah R, Zhang X, Talapatra S. Electrochemical double layer capacitor electrodes using aligned carbon nanotubes grown directly on metals. Nanotechnology. 2009;20:395202.

18. Bistamam MSA, Azam MA. Tip-growth of aligned carbon nanotubes on cobalt catalyst supported by alumina using alcohol catalytic chemical vapor deposition. Results Phys. 2014;4:105-106.

19. Zhang H, Cao G, Wang Z, et al. Electrochemical capacitive properties of carbon nanotube arrays directly grown on glassy carbon and tantalum foils. Carbon. 2008;46:818-832.

20. Gao L, Peng A, Wang ZY, et al. Growth of aligned carbon nanotube arrays on metallic substrate and its application to supercapacitors. Solid State Commun. 2008;146:380-383.

21. Kim B, Chung H, Min BK, et al. Electrochemical Capacitors Based on Aligned Carbon Nanotubes Directly Synthesized on Tantalum Substrates. Bull Kor Chem Soc. 2010;31:3697-3702.

22. Azam MA, Azizan MA, Manaf NSA, et al. Electrode Fabrication and Electrochemical Analysis of AC/Graphene-Based Electrochemical. Adv Sci Eng Med. 2014;6:1-4.

23. Azam MA, Isomura K, Fujiwara A, et al. Direct growth of vertically aligned single-walled carbon nanotubes on conducting substrate and its electrochemical performance in ionic liquids. Phys Status Solidi A. 2012;209:2260-2266.

24. Chen J, Li W, Wang D, et al. Electrochemical characterization of carbon nanotubes as electrode in electrochemical double-layer capacitors. Carbon. 2002;40:1193-1197.

25. Fan LZ, Maier J. High-performance polypyrrole electrode materials for redox supercapacitors. Electrochem Commun. 2006;8:937-940.

26. Kim B, Chung H, Kim W. High-performance supercapacitors based on vertically aligned carbon nanotubes and nonaqueous electrolytes. Nanotechnology. 2012;23:155401. 
27. Frackowiak E. Carbon materials for supercapacitor application. Phys Chem Chem Phys. 2007;9:1774-1785.

28. Yan J, Liu J, Fan Z, et al. High-performance supercapacitor electrodes based on highly corrugated graphene sheets. Carbon. 2012;50:2179-2188.

29. Han $\mathrm{G}$, Liu $\mathrm{Y}$, Zhang $\mathrm{L}$, et al. $\mathrm{MnO}_{2}$ nanorods intercalating graphene oxide/polyaniline ternary composites for robust high-performance supercapacitors. Sci Rep. 2014;4:4824.

30. Azam MA, Jantan NH, Dorah N, et al. Activated carbon and single-walled carbon nanotube based electrochemical capacitor in $1 \mathrm{M} \mathrm{LiPF} 6$ electrolyte. Mater Res Bull. 2015;69:20-23.

31. Farma R, Deraman M, Awitdrus, et al. Physical and Electrochemical Properties of Supercapacitor Electrodes Derived from Carbon Nanotube and Biomass Carbon. Int J Electrochem Sci. 2013;8:257-273.

32. Qiu Y, Li G, Hou Y, et al. Chem Mater. 2015;27:1194-1200. 